\title{
INFLUÊNCIA DA ATIVIDADE FÍSICA SOBRE A QUALIDADE DE VIDA DE GESTANTES: UM ESTUDO TRANSVERSAL
}

\section{INFLUENCE OF PHYSICAL ACTIVITY ON THE QUALITY OF LIFE IN PREGNANTS: A CROSS STUDY}

\author{
Jôse Maryane Lima*, Waldinéia Bispo*, André Luiz Cordeiro**
}

\author{
Autora para correspondência: André Luiz Cordeiro - andrelisboacordeiro@gmail.com \\ *Graduanda em Fisioterapia pela Faculdade Nobre, Feira de Santana - Bahia \\ **Mestrando em Medicina e Saúde Humana pela Escola Bahiana de Medicina e Saúde Pública, Salvador - \\ Bahia. Docente da Faculdade Nobre, Feira de Santana - Bahia
}

\section{R E S U M O}

\begin{abstract}
Introdução: A gestação provoca alterações que incidem principalmente nos sistemas cardiorrespiratório, musculoesquelético e no metabolismo geral que não se restringe apenas aos órgãos, mas também à mecânica do corpo feminino. Além de atentar para todas essas alterações, é necessário que os profissionais de saúde preocupem-se com a qualidade de vida dessas mulheres, o que trará benefícios tanto para ela como também para o feto. Objetivos: Avaliar o impacto do exercício físico sobre a qualidade de vida em gestantes. Metodologia: Trata-se de um estudo transversal realizado com gestantes. Elas responderam a um questionário internacional de atividade física, intitulado IPAQ (International Physical Activity Questionnaire) versão curta e divididas em sedentárias e fisicamente ativas. Além disso, um questionário de qualidade de vida (SF - 36). Resultados: Foram avaliadas 56 mulheres, tendo as grupo "Ativa" média de idade de 28,6 $\pm 5,6$ anos e grupo "Sedentária" a média de idade foi de $29 \pm 6$ anos. No quesito dor o grupo "Ativa" teve uma melhor pontuação quando comparado ao grupo "Sedentário" (48,4 \pm 29 vs. $69 \pm 23,7$, respectivamente ( $p=0,007)$. O mesmo aconteceu com Estado Geral da Saúde $50 \pm 11,5$ vs. 57,1 \pm 10,6 ( $p=0,01)$ e Limitação por Aspectos Emocionais 38,2 $\pm 9,9$ vs. 54,5 $\pm 14,1(p<0,001)$. Conclusão: Com base nos achados do presente estudo, o exercício físico influencia positivamente a qualidade de vida das gestantes.
\end{abstract}

Palavras-chaves: Exercício; Gravidez; Qualidade de vida. 
Introduction: Pregnancy causes changes mainly in cardiorespiratory systems, musculoskeletal and overall metabolism that is not restricted to the organs, but also the mechanics of the female body. In addition to pay attention to all these changes, it is necessary that health professionals worry with the quality of life of these women, which will bring benefits both to them and also to the fetus. Objetive: To assess the impact of exercise on quality of life in pregnant women. Methodology: This is a cross-sectional study of pregnant women. They responded to an international physical activity questionnaire, entitled IPAQ (International Physical Activity Questionnaire) short version and divided into sedentary and physically active Also, to a questionnaire of quality of life (SF - 36). Results: 56 women were evaluated. The group "Active" has a mean age of $28.6 \pm 5.6$ years and in the group "Sedentary" the average age was 29 \pm 6 years. On the issue of pain the group "Active" scored better when compared to "Sedentary" (48.4 \pm 29 vs. $69 \pm 23.7$, respectively $(p=0.007$ ). So did General Health $50 \pm 11.5$ vs. $57.1 \pm 10.6$ ( $p=$ 0.01 ) and Emotional Aspects Limitation $38.2 \pm 9.9$ vs. $54.5 \pm 14.1$ ( $p<0.001$ ). Conclusion: Based on the findings of this study, exercise positively influences the quality of life of pregnant women.

Keywords: Exercise; Pregnancy; Quality of life. 


\section{INTRODUÇÃO}

Um momento único na vida de qualquer mulher é a gravidez, momento este que proporciona diversas modificações e alterações no organismo materno, gerando diversas adaptações fisiológicas, que geralmente são atribuídas a mudanças hormonais, aumento do útero e de tecidos, mudanças essas necessárias para a adaptação e crescimento do embrião e depois do feto durante o período gravídico'.

Além de atentar para todas essas alterações, é necessário que os profissionais de saúde preocupemse com a qualidade de vida dessas mulheres, o que trará benefícios tanto para ela como também para - feto. Conforme Rezende e Souza ${ }^{2}$ é importante - reconhecimento dos fatores desconfortantes que podem influenciar negativamente na gestação e, consequentemente, na qualidade de vida.

A avaliação da qualidade de vida torna-se importante instrumento de gestão do cuidado, podendo através de uma avaliação modificar uma dada situação, diagnosticando uma realidade a fim de nela intervir. Dessa forma, a avaliação da qualidade de vida pode ajudar a dimensionar e analisar as ações de promoção da saúde, que poderiam ser implantadas nas comunidades e nos programas de saúde ${ }^{3}$.

Para melhora da qualidade de vida das gestantes devem ser feitas intervenções com a finalidade de controlar $\circ$ peso, as algias posturais, o estresse, a diminuição do risco de depressão puerperal, a maior autonomia no trabalho de parto ${ }^{4}$.

Nesse contexto de promoção de saúde e qualidade de vida destaca-se a prática de exercícios físicos. Conforme Batista e colaboradores ${ }^{5}$ a atividade física é definida como qualquer movimento corporal decorrente de contração muscular, com dispêndio energético acima do repouso que, em última análise, permite o aumento da força física, flexibilidade do corpo e maior resistência, com mudanças, seja no campo da composição corporal ou de desempenho desportiva.

Assim, a opção por um estilo de vida mais saudável contribui para que as gestantes tornem-se mais autoconfiantes e satisfeitas com a aparência. Porém, apesar de todos os benefícios, a adesão ao exercício durante a gestação ainda é difícil, pois muitas mulheres têm receios quanto à segurança da sua prática ${ }^{4}$. Por isso que $\circ$ papel dos profissionais de saúde é extremamente importante nos esclarecimentos de dúvidas e mitos, encorajando a gestante a se exercitar.

A atividade física aeróbia contribui de forma bastante considerável no controle do peso e condicionamento físico, reduzir os riscos de diabetes gestacional. Ao ativar grupos musculares a glicose é bem utilizada assim aumenta sensibilidade à insulina. A prática de atividade frequente apresenta fatores protetores à saúde mental psicológica durante $\circ$ período gestacional e após.

Baseado nisso, o objetivo desse trabalho foi avaliar o impacto do exercício físico sobre a qualidade de vida em gestantes.

\section{MÉTODOS}

Trata-se de um estudo transversal realizado em clínicas e academias da cidade de Feira de Santana - Bahia durante o período de julho a setembro de 2016. A pesquisa foi realizada com 56 mulheres de acordo com escolha intencional. Usando como critérios de inclusão mulheres acima de 18 anos, com tempo de gestação acima de 3 meses, que praticaram ou não exercício físico durante a gravidez. E como critérios de exclusão mulheres que não tinham discernimento para responder aos questionários ou que não aceitaram assinar o termo de consentimento livre e esclarecido.

Para coletar os dados sobre o público-alvo do estudo e descrever os aspectos que envolvem as gestantes, foi executado um questionário aberto, com as variáveis envolvendo a identificação da gestante e história ginecológica (quantidade de filhos, tipo de parto e se realizou episiotomia). 
Após esse momento elas responderam a um questionário internacional de atividade física, intitulado IPAQ (International Physical Activity Questionnaire) versão curta é um questionário que permite estimar o tempo semanal gasto em atividades físicas de intensidade moderada e vigorosa, em diferentes contextos do cotidiano, como: trabalho, transporte, tarefas domésticas e lazer, e ainda o tempo despendido em atividades passivas, realizadas na posição sentada.

De acordo com a reposta das participantes as mesmas foram divididas em sedentárias (Não realiza nenhuma atividade física por pelo menos 10 minutos contínuos durante a semana) ou fisicamente ativas (atividade física vigorosa $\geq 3$ dias/semana e $\geq 20$ minutos / sessão; moderada ou caminhada - $\geq 5$ dias/semana e $\geq 30$ minutos/sessão; qualquer atividade somada: $\geq 5$ dias / semana e $\geq$ $150 \mathrm{~min} /$ semana) 6 .
Além disso, um questionário de qualidade de vida (SF - 36) foi aplicado para todas as gestantes, questionário este simples, breve e auto administrável no qual se as participantes avaliam à sua saúde, a forma como se sentem e sua capacidade de desempenho nas atividades habituais. Desta forma, realizou-se um comparativo, buscando identificar qual dos dois grupos possuem uma melhor qualidade de vida.

A análise de dados foi expressa em média e desvio padrão. Para análise de normalidades, foi utilizado o teste de Kolmogorov-Smirnov, para comparação entre grupos utilizou-se o teste $\mathrm{T}$ de Student não pareado, e, para análise das variáveis categóricas, o teste de Qui quadrado. Foi considerado como significativo um $p$ menor que 0,05. $O$ trabalho foi aprovado pelo Comitê de Ética e Pesquisa da Faculdade Nobre (CEP-FAN) de Feira de Santana, sob CAAE 49432315.6.0000.5654.

\section{RESULTADOS}

Em relação à avaliação do questionário de qualidade de vida MINICHAL-BRASIL, pode ser observado que através da média obtida das questões, de acordo com a escala de Likert, que as questões que abrangiam o estado mental, foram as mais assinaladas negativamente (média 6,35).

Tabela 1. Características das gestantes avaliadas.

\begin{tabular}{|c|c|c|c|}
\hline Variáveis & $\begin{array}{l}\text { Sedentárias } \\
(n=25)\end{array}$ & $\begin{array}{l}\text { Ativas } \\
(n=31)\end{array}$ & $p$ \\
\hline \multicolumn{4}{|l|}{ Gênero } \\
\hline Feminino & $25(100 \%)$ & $25(100 \%)$ & \\
\hline Idade & $29 \pm 6$ & $28,6 \pm 5,6$ & $0,82 a$ \\
\hline Número de Filhos & $1 \pm 1,2$ & $0,7 \pm 0,7$ & $0,43 a$ \\
\hline Estado Civil & & & $0,52 b$ \\
\hline Solteira & $3(12 \%)$ & $5(16,1 \%)$ & \\
\hline Casada & $8(32 \%)$ & $14(45,1 \%)$ & \\
\hline Viúva & $3(12 \%)$ & $2(6,5 \%)$ & \\
\hline União Estável & $11(44 \%)$ & $10(32,3 \%)$ & \\
\hline Raça & & & $0,02 b$ \\
\hline Branca & $3(8,6 \%)$ & $10(32,3 \%)$ & \\
\hline Negra & $5(23,4 \%)$ & $9(29 \%)$ & \\
\hline Parda & $17(68 \%)$ & $12(38,7 \%)$ & \\
\hline
\end{tabular}

a Teste T de Student independente; $b$ Teste de ANOVA. 
Após a resposta do questionário SF-36 foi calculada a média e o desvio padrão das diferentes variáveis em cada grupo. Notou-se alguns componentes apresentaram diferença na comparação entre os grupos, como dor ( $p=0,007$ ), estado geral da saúde $(p=0,01)$ e limitação por aspectos emocionais $(p<0,001)$. As demais variáveis apresentaram melhores valores no grupo "Ativas", porém sem significância estatística. A tabela 2 demonstra todos os componentes analisados.

Tabela 2. Análise da diferença da qualidade de vida das gestantes avaliados através do SF-36.

\begin{tabular}{llcc}
\hline Variáveis & $\begin{array}{c}\text { Sedentárias } \\
(\mathbf{n}=\mathbf{2 5})\end{array}$ & $\begin{array}{c}\text { Ativas } \\
(\mathbf{n}=31)\end{array}$ & $\mathbf{p}$ \\
\hline Capacidade Funcional & $60 \pm 26,7$ & $66,1 \pm 24,6$ & 0,41 \\
$\begin{array}{l}\text { Limitação por Aspectos } \\
\text { Físicos }\end{array}$ & $42 \pm 40,5$ & $59 \pm 43,6$ & 0,11 \\
Dor & $48,4 \pm 29$ & $69 \pm 23,7$ & 0,01 \\
Estado Geral da Saúde & $50 \pm 11,5$ & $57,1 \pm 10,6$ & 0,01 \\
Vitalidade & $52,1 \pm 15,3$ & $59,7 \pm 3,3$ & 0,06 \\
Aspectos Sociais & $48 \pm 12,3$ & $52,1 \pm 11,6$ & 0,31 \\
Limitação Por Aspectos & $38,2 \pm 9,9$ & & $<0,001$ \\
Emocionais & $79,4 \pm 24,6$ & $54,5 \pm 14,1$ & 0,06 \\
\hline Saúde Mental & & $91,5 \pm 5,2$ & \\
\hline
\end{tabular}

\section{DISCUSSÃO}

No presente estudo ficou exposto que a atividade física influencia positivamente a qualidade de vida das gestantes principalmente no tocante dor, estado geral da saúde e limitação por aspectos emocionais. As demais variáveis também apresentaram melhores resultados no grupo ativa, porém sem significância estatística.

Assim, as gestantes que praticaram atividades físicas sentiram menos dores quando comparadas com aquelas gestantes sedentárias. Resultados similares foram encontrados por Sebben e colaboradores ${ }^{7}$, no qual as gestantes relataram a ocorrência de dor em região lombo-sacra, surgindo entre a $16^{a}$ e $26^{a}$ semana de gestação e que a fisioterapia, através do tratamento hidroterapêutico, mos $\neg$ trouse altamente eficaz na redução do quadro álgico lombar.

Segundo Surita e colaboradores ${ }^{8}$ na gestação a adesão ao exercício ainda é difícil, pois muitas mulheres têm receios e dúvidas quanto à segurança da sua prática, necessitando de esclarecimentos e incentivos constantes, que precisam ser feitos pelos profissionais que as atendem durante o pré-natal.

Em um estudo foi demonstrado que o nível de atividade física diminui com o avançar do tempo gestacional. Na trigésima segunda semana, 100\% das mulheres foram consideradas sedentárias, com diminuição importante das atividades domesticas e laborais. Esse resultado pode estar relacionado com o presente estudo o qual verificou que a capacidade funcional era menor no grupo inativo?

Tendais e colaboradores ${ }^{10}$ utilizaram $\circ$ SF-36 para avaliar a qualidade de vida de gestantes e observaram que existe uma diminuição independentemente do nível de atividade, porém a função mental não sofre alteração. $O$ que foi 
visto no presente estudo foi um valor mais benéfico, em todas as variáveis, nas gestantes que eram fisicamente ativas sem ser possível afirmar que existiu uma melhora pois não houve uma avaliação e comparação com o período pré-gestação.

Em um estudo qualitativo sobre a alteração da atividade física durante a gestação verificando que quase todas as mulheres alteraram o seu padrão de atividade nesse período de forma a adaptar-se tanto psicológica quando fisiologicamente ${ }^{11}$.

No presente estudo ficou exposto que a saúde mental foi melhor no grupo que realizava atividade física, resultado semelhante foi encontrado no trabalho de Daley e colaboradores ${ }^{12}$ que avaliaram 208 mulheres que praticavam atividade e tiveram uma incidência menor de depressão quando comparadas as sedentárias. Já Vallim e colaboradores ${ }^{13}$ utilizaram um programa de exercícios aeróbicos na piscina para avaliar a qualidade de vida das gestantes. Para isso eles utilizaram uma ferramenta que foi a WHOQOL-BREF que tem a mesma função do SF-36. E, diferente dos resultados do presente estudo eles concluíram que o exercício físico não influencia na qualidade de vida desses indivíduos. Ainda nesse sentido, Nascimento e colaboradores ${ }^{4}$ afirmam em seu ensaio clínico randomizado que - exercício físico não influencia na percepção de qualidade de vida das gestantes, novamente não corroborando os dados do presente estudo.

Dentre as limitações desse trabalho pode-se destacar a falta de um calculo amostral, a não avaliação do tipo de exercício e tempo que essa gestante pratica.

\section{CONCLUSÃO}

Com base nos achados do presente estudo, o exercício físico influencia positivamente a qualidade de vida das gestantes. Novos trabalhos são necessários visando a realização de atividade física com protocolo controlado para confirmação de todos os possíveis benefícios do exercício nessa população.

\section{REFERÊNCIAS}

1. Canesin KF, Amaral WN. Atuação

fisioterapêutica para diminuição do tempo do trabalho de parto: revisão de literatura. FEMINA. 2010;38(8):1-10.

2. Rezende CL, Souza JC. Qualidade de vida das gestantes de alto risco de um centro de atendimento à mulher. Psicólogo informação. 2012;16(16):45-69.

3. Castro DFA, Fracolli LA. Qualidade de vida e promoção da saúde: em foco as gestantes. $O$ Mundo da Saúde. 2013;37(2):159-165.

4. Nascimento SL, Surita FG, Parpinelli MA, Siani S, Pinto, Silva JL. The effect of an antenatal physical exercise programme on maternal/perinatal outcomes and quality of life in overweight and obese pregnant women: a randomised clinical trial. BJOG. 2011 ; 118 (12): 1455-1463. doi: $10.1111 /$ j.1471-0528.2011.03084.x

5. Batista DC, Chiara VL, Gugelmin SA, Martins PD. Atividade física e gestação: saúde da gestante não atleta e crescimento fetal. Rev. Bras. Saúde Matern. Infant. 2003;3(2):151-158. doi: 10.1590/ S1519-38292003000200004

6. Silva FT. Avaliação do nível de atividade física durante a gestação. Rev Bras Ginecol Obstet. 2007;29(9):490-500. doi: 10.1590/S010072032007000900009

7. Sebben V, Perussolo C, Guedes JM, Tourinho HF. Tratamento hidroterapêutico na dor lombar em gestantes. Perspectiva. 2011 ;35(129):167-175.

8. Surita FG, Nascimento SL, Silva JLP. Exercício físico e gestação. Rev Bras Ginecol Obstet. 2014;36(12):531-4. doi: 10.1590/SO100720320140005176

9. Tavares JS, Melo AS, Amorim MM, Barros VO, Takito MY, Benício MH et al. Padrão de atividade física entre gestantes atendidas pela estratégia saúde da família de Campina Grande - PB. Rev Bras Epidemiol. 2009;1 2(1):10-9. doi: 10.1590/ S1415-790X2009000100002 
10. Tendais I, Figueiredo B, Mota J, Conde A.

Physical activity, health-related quality of life and depression during pregnancy. Cad. Saúde Pública. 2011 ;27(2):219:228. doi: 10.1590/S0102$311 \times 2011000200003$

11. Hegaard HK, Kiaergaard H, Damm PP, Petersson K, Dykes AK. Experiences of physical activity during pregnancy in Danish nulliparous women with a physically active life before pregnancy. A qualitative study. BMC Pregnancy and Childbirth. 2010;10(30):1-10. doi: $10.1186 / 1471-2393-10-33$

12. Daley AJ, Jolly K, Sharp DJ, Turner KM, Blamey RV, Coleman $S$ et al. The effectiveness of exercise as a treatment for postnatal depression: study protocol. Pregnancy and Childbirth.

2012;12(45):1-8. doi: 10.1 186/1471-2393-1245

13. Vallim AL, Osis MJ, Cecatti JG, Baciuk EP, Silveira C, Cavalcante SR. Water exercises and quality of life during pregnancy. Reproductive Health. 2011 ;8(14):1-7. doi: 10.1 186/17424755-8-14 